\title{
Retrospective Analysis of Anterior Interosseous Nerve Lesions and Motor Nerve Conduction Measurements
}

\author{
Shingo Nobuta ${ }^{1 *}$, Katsumi Sato ${ }^{2}$ and Eiji Itoi ${ }^{3}$ \\ ${ }^{1}$ Department of Orthopaedic Surgery, Tohoku Rosai Hospital, Sendai, Japan \\ ${ }^{2}$ Tohoku Rosai Hospital, Sendai, Japan \\ ${ }^{3}$ Department of Orthopaedic Surgery, Tohoku University School of Medicine, Sendai, Japan
}

\begin{abstract}
Objective: Anterior interosseous nerve (AIN) lesions are rare and a few nerve conduction measurements have been reported. The purpose of this retrospective study was to assess the diagnostic value of the motor nerve conduction measurements for the AIN lesion and to investigate its electrodiagnostic features.
\end{abstract}

Methods: Twenty-three patients with AIN lesion were reviewed after a mean follow-up of 13 months. Of these 23 cases, a complete palsy of flexor pollicis longus (FPL) and flexor digitorum profundus to the index finger (FDP1) was seen in five, and an isolated palsy of FPL in three and that of FDP1 in two, while pronator quadratus (PQ) was weak in twelve. The compound muscle action potential (CMAP) from the PQ muscle and the FPL muscle were recorded and analyzed. Twenty-two patients were treated conservatively and one underwent surgery.

Results: Both PQ-CMAP and FPL-CMAP were recorded in all of the patients initially, and they all showed abnormality in PQ- and/or FPL-CMAP. Delayed latency and / or low amplitude were seen in 21 for PQ-CMAP and in 17 for FPL-CMAP. At the time of final follow-up, an improvement in the muscle strength of FPL, FDP1 and PQ was seen in 11 with the British Medical Research Council (MRC) grade 4 or better (complete recovery), whereas, partial recovery in 11 and unchanged in one. The decrease of amplitude in PQ-CMAP showed a significant difference between the normal $P Q$ strength group and the weak $P Q$ strength group.

Conclusion: Recording and analysis of PQ- and FPL-CMAP are simple and safe and are important for the definite electrodiagnosis of AIN lesions. The data suggest that an axonal degeneration is main pathology.

Keywords: Anterior interosseous nerve; Nerve conduction measurement; Compound muscle action potential; Pronator quadratus; Flexor pollicis longus; Brachial plexus neuritis; Conservative treatment

\section{Introduction}

The anterior interosseous nerve (AIN) lesion remains a rare lesion. Clinical features of the AIN lesion are paralyis of flexor pollicis longus (FPL), flexor digitorum profundus to the index finger (FDP1) and pronator quadratus $(\mathrm{PQ})$, while the thenar muscle atrophy and sensory complaints of the median nerve distribution are absent. The AIN lesion was described in association with brachial neuritis by Parsonage and Turner [1] in 1948, and was later identified by Kiloh and Nevin [2] in 1952. Fearn and Goodfellow [3] were the first to do surgical exploration for the diagnosis and treatment. For electrodiagnosis of the AIN lesions, many previous reports described that the electromyogram revealed fibrillation or sharp wave, or neurogenic recruitment in innervated muscles [4-15]. However, there are a few nerve conduction measurements for AIN lesions reported up to the present [16-23]. We reported the importance of nerve conduction measurement at AIN lesions for ten patients in 2008 [24]. The purpose of the present retrospective study was to assess the diagnostic value of the motor nerve conduction measurements by recording the compound muscle action potential (CMAP) from the PQ and FPL at the AIN lesion and to investigate its electrodiagnostic features.

\section{Patients and Methods}

Between 2004 and 2014, 23 patients with spontaneous and nontraumatic AIN lesion were reviewed after a mean follow-up of 13 months (range, 6-51). There were 14 males and nine females, with a mean age of 46 years (range, 22-70). Affected side was right in nine and left in 14. Pain around the elbow, forearm, upper arm or shoulder on the ipsilateral side was present in 20 patients and lasted for three days to two months (mean, 20 days) before the onset of paralysis. A complete palsy of FPL and FDP1 was seen in five, and an isolated palsy of FPL in three and that of FDP1 in two and meanwhile PQ was weak in 12. PQ was tested with the elbow in a full flexed position to neutralize the humeral head of the pronator teres muscle. There was no sensory loss in the affected upper limb and shoulder girdle strength was intact, and flexor carpi radialis, pronator teres (with the elbow extended position), and palmaris longus were intact in all patients. One of the authors (S. Nobuta) and an other occupational therapist tested the muscle strength. The duration of paralysis was one to seven months (mean, 3 months). CMAP from the abductor pollicis brevis (APB) was recorded to indicate median nerve conduction measurements, revealing normal latency and amplitude in all patients. The details of the patients are shown in Table 1. Twenty-two patients were treated conservatively, and one underwent neurolysis surgery seven months after the onset of palsy (case 7).

Informed consent was obtained from all patients. Motor nerve conduction measurements were performed in all patients before treatment and at the time of the final follow-up. Patients were examined sitting in a chair with the elbow extended and pronated (recording

*Corresponding author: Shingo Nobuta, Department of Orthopaedic Surgery, Tohoku Rosai Hospital, 4-3-21, Dainohara, Aoba-ku, Sendai, Miyagi 981-8563, Japan, Tel: +81-22-275-1111; Fax: +81-22-275-7541; E-mail: nobutays@jc5.so-net.ne.jp

Received December 15, 2016; Accepted January 20, 2017; Published January 27, 2017

Citation: Nobuta S, Sato K, Itoi E (2017) Retrospective Analysis of Anterior Interosseous Nerve Lesions and Motor Nerve Conduction Measurements. J Neurol Neurophysiol 8: 411. doi:10.4172/2155-9562.1000411

Copyright: $\odot 2017$ Nobuta S, et al. This is an open-access article distributed under the terms of the Creative Commons Attribution License, which permits unrestricted use, distribution, and reproduction in any medium, provided the original author and source are credited. 
Citation: Nobuta S, Sato K, Itoi E (2017) Retrospective Analysis of Anterior Interosseous Nerve Lesions and Motor Nerve Conduction Measurements. J Neurol Neurophysiol 8: 411. doi:10.4172/2155-9562.1000411

Page 2 of 5

\begin{tabular}{|c|c|c|c|c|c|c|c|c|c|c|c|c|c|c|}
\hline \multirow[b]{3}{*}{ Age } & \multirow{3}{*}{\begin{tabular}{|l|} 
Case \\
Gender
\end{tabular}} & \multirow[b]{3}{*}{ Side } & \multirow{3}{*}{$\begin{array}{l}\text { Site of pain } \\
\text { (duration) }\end{array}$} & \multirow{3}{*}{$\begin{array}{l}\text { Duration of } \\
\text { paralysis }\end{array}$} & \multirow{2}{*}{\multicolumn{3}{|c|}{ MRC }} & \multicolumn{4}{|c|}{$\begin{array}{l}\text { Parameter measurements before/after } \\
\text { treatment }\end{array}$} & \multirow{3}{*}{$\begin{array}{c}\begin{array}{c}\text { Onset } \\
\text { recovery }\end{array} \\
\text { Mo }\end{array}$} & \multirow{3}{*}{\begin{tabular}{|c|}
$\begin{array}{c}\text { Follow } \\
\text {-up }\end{array}$ \\
Mo
\end{tabular}} & \multirow{3}{*}{ Result } \\
\hline & & & & & & & & \multicolumn{2}{|c|}{ PQ-CMAP } & \multicolumn{2}{|c|}{ FPL-CMAP } & & & \\
\hline & & & & & FPL & FDP1 & $P Q$ & Lat. & Amp. & Lat. & Amp. & & & \\
\hline 1. 42 & M & $\mathrm{R}$ & $\mathrm{UA}(1 \mathrm{~m})$ & $2 m$ & 5 & 3 & 5 & $4.1 / 3.7$ & $\underline{3.8 / 2.6}$ & $\underline{4.1 / 4.0}$ & $\underline{6.4} \underline{6.1}$ & 3 & 9 & C \\
\hline 2. 58 & $\mathrm{~F}$ & $\mathrm{R}$ & $\mathrm{FA}(2 \mathrm{w})$ & $3 \mathrm{~m}$ & 0 & 0 & 2 & $3.2 / 2.6$ & $0.8 / 0.9$ & $\underline{3.6 / 3.4}$ & $\underline{2.1 / 2.2}$ & 4 & 6 & $P$ \\
\hline 3. 49 & $\mathrm{~F}$ & L & $\mathrm{FA}(2 \mathrm{w})$ & $2 \mathrm{~m}$ & 3 & 3 & 5 & $3.6 / \underline{3.0}$ & $0.6 / 1.1$ & $4.2 / 4.7$ & $0.7 / 0.9$ & 5 & 11 & C \\
\hline 4. 56 & M & L & None & $6 \mathrm{~m}$ & 1 & 0 & 5 & $\underline{3.8 / 3.4}$ & $\underline{0.6 / 0.7}$ & $\underline{3.8 / 4.0}$ & $1.7 / \underline{2.2}$ & 7 & 12 & $\mathrm{P}$ \\
\hline 5. 33 & $\mathrm{~F}$ & L & $E(3 w)$ & $2 m$ & 4 & 1 & 5 & $\underline{2.7 / 2.6}$ & $\overline{1.8 / 2.2}$ & $3.8 / 3.7$ & $4.5 / 4.5$ & & 12 & u \\
\hline 6. 34 & $M$ & L & $\mathrm{UA}(1 \mathrm{w})$ & $1 \mathrm{~m}$ & 1 & 5 & 5 & $3.6 / \underline{3.0}$ & $2.0 / 2.2$ & $\underline{4.2} / \underline{4.0}$ & $2.8 / 5.0$ & 8 & 18 & $P$ \\
\hline 7. 22 & M & $\mathrm{R}$ & $\mathrm{E}(1 \mathrm{~m})$ & $7 \mathrm{~m}$ & 0 & 0 & 3 & $3.0 / 3.4$ & $0.7 / 0.7$ & $\underline{3.8 / 4.1}$ & $2.2 / 1.9$ & 8 & 12 & $\mathrm{P}$ \\
\hline 8. 28 & M & L & UA $(4 \mathrm{w})$ & $4 \mathrm{~m}$ & 0 & 5 & 5 & $\underline{3.1} \underline{3.2}$ & $\underline{1.3 / 2.3}$ & $4.1 / 3.8$ & $4.9 / \underline{5.7}$ & 5 & 7 & $P$ \\
\hline 9. 63 & $M$ & L & $S(2 w)$ & $7 \mathrm{~m}$ & 0 & 0 & 5 & $\underline{3.7} / \underline{3.7}$ & $1.6 / \underline{1.8}$ & $\underline{4.5} \underline{4.5}$ & $\underline{3.5} / \underline{4.5}$ & 9 & 13 & $P$ \\
\hline 10. 31 & $M$ & $L$ & None & $1 \mathrm{~m}$ & 2 & 2 & 4 & $6.4 / 3.0$ & $0.3 / 0.5$ & $8.3 / 4.5$ & $3.4 / 1.8$ & 3 & 6 & C \\
\hline 11.70 & $M$ & $R$ & $\mathrm{E}(2 \mathrm{~m})$ & $2 \mathrm{~m}$ & 2 & 0 & 3 & $4.9 / 4.5$ & $\underline{0.8 / 1.2}$ & $5.1 / 4.5$ & $\underline{0.4 / 1.8}$ & 3 & 13 & C \\
\hline 12. 42 & $M$ & L & $\mathrm{FA}(2 \mathrm{w})$ & $3 \mathrm{~m}$ & 0 & 0 & 3 & $3.5 / 3.4$ & $1.1 / 1.2$ & $4.3 / 3.9$ & $\underline{5.1} \underline{15.2}$ & 10 & 21 & C \\
\hline 13. 45 & $\mathrm{~F}$ & L & $\mathrm{UA}(2 \mathrm{w})$ & $2 \mathrm{~m}$ & 4 & 1 & 2 & $4.0 / 3.8$ & $0.5 / 1.0$ & $\underline{3.9} / \underline{3.7}$ & $3.7 / 3.4$ & 6 & 8 & $P$ \\
\hline 14. 50 & $\mathrm{~F}$ & L & $\mathrm{UA}(3 \mathrm{~d})$ & $1 \mathrm{~m}$ & 3 & 2 & 5 & $4.0 / \underline{2.7}$ & $0.3 / 1.7$ & $4.1 / 3.7$ & $1.5 / \underline{4.0}$ & 2 & 6 & C \\
\hline 15. 38 & M & $R$ & $\mathrm{~S}(6 \mathrm{w})$ & $3 \mathrm{~m}$ & 0 & 2 & 3 & $3.5 / 3.5$ & $1.1 / 1.5$ & $4.4 / \underline{4.3}$ & $4.2 / 3.9$ & 6 & 8 & C \\
\hline 16. 67 & $\mathrm{~F}$ & $L$ & $\mathrm{~S}(4 \mathrm{w})$ & $7 \mathrm{~m}$ & 1 & 1 & 2 & $\underline{3.0} / \underline{3.0}$ & $1.0 / 0.7$ & $\underline{4.0} \underline{\underline{3.7}}$ & $2.0 / 2.3$ & 9 & 20 & $P$ \\
\hline 17. 69 & $\mathrm{~F}$ & $R$ & $\mathrm{E}(4 \mathrm{w})$ & $7 \mathrm{~m}$ & 4 & 2 & 4 & $3.5 / 3.0$ & $0.8 / 1.2$ & $3.7 / 3.7$ & $2.8 / 3.0$ & 13 & 19 & $P$ \\
\hline 18. 42 & $M$ & L & None & $5 \mathrm{~m}$ & 5 & 2 & 5 & $\underline{3.0 / 3.8}$ & $1.4 / 1.9$ & $\underline{4.0 / 4.2}$ & $\underline{3.5} / 2.8$ & 6 & 10 & C \\
\hline 19. 33 & $M$ & $\mathrm{~L}$ & $E(2 w)$ & $1 \mathrm{~m}$ & 2 & 2 & 4 & $3.5 / \underline{3.3}$ & $\underline{3.2} \underline{3.3}$ & $\underline{4.3} / 4.5$ & $\underline{3.2 / 2.1}$ & 2 & 6 & C \\
\hline 20. 59 & $\mathrm{~F}$ & $\mathrm{R}$ & $E(10 d)$ & $1 \mathrm{~m}$ & 0 & 2 & 2 & $3.4 / 3.2$ & $\overline{1.0 / 1.6}$ & $3.5 / 3.7$ & $3.1 / 3.2$ & 10 & 51 & C \\
\hline 21. 43 & $M$ & $\mathrm{R}$ & $S(2 w)$ & $1 \mathrm{~m}$ & 0 & 0 & 5 & $3.3 / 3.2$ & $1.4 / 2.1$ & $4.6 / \underline{4.0}$ & $2.5 / 5.0$ & 10 & 18 & $P$ \\
\hline 22. 55 & $\mathrm{~F}$ & $L$ & $E(2 w)$ & $1 \mathrm{~m}$ & 0 & 1 & 3 & $3.9 / 3.2$ & $1.7 / 1.7$ & $\underline{3.8 / 3.5}$ & $5.4 / 4.2$ & 4 & 13 & $P$ \\
\hline 23. 27 & M & $R$ & $E(3 d)$ & $1 \mathrm{~m}$ & 2 & 5 & 5 & $\underline{3.6} / \underline{3.6}$ & $1.6 / 1.6$ & $\underline{4.2 / 4.2}$ & $6.2 / 6.9$ & 2 & 6 & C \\
\hline
\end{tabular}

Table 1: Details of 23 cases with the anterior interosseous nerve lesion.

M: Male; F: Female; R: Right; L: Left; UA: Upper Arm; FA: Forearm; E: Elbow; S: Shoulder; m: Month(s); w: Week(s); d: Day(s); MRC: Initial Evaluation of Medical Research Council grade; FPL: Flexor Pollicis Longus; FDP1: Flexor Digitorum Profundus to the Index Finger; PQ: Pronator Quadratus; CMAP: Compound Muscle Action Potential; Lat.: Latency (msec); Amp.: Amplitude (mV), Mo: Months; C: Complete Recovery (MRC grade 4 or better); P: Partial Recovery, U: Unchanged; : Side-to-Side Difference is in the Normal Range

\begin{tabular}{|c|l|c|c|c|c|}
\hline \multicolumn{2}{|c|}{} & Right & Left & $\begin{array}{c}\text { Side-to-side } \\
\text { difference }\end{array}$ & Mean 2 SD \\
\hline \multirow{2}{*}{ PQ-CMAP } & Latency (SD) & $3.47(0.35)$ & $3.47(0.34)$ & $0.07(0.07)$ & $0.20 \mathrm{~ms}$ \\
\cline { 2 - 6 } & Amp. (SD) & $4.44(1.17)$ & $4.29(1.11)$ & $0.68(0.47)$ & $1.61 \mathrm{mV}$ \\
\hline \multirow{2}{*}{ FPL-CMAP } & Latency (SD) & $3.89(0.32)$ & $3.90(0.31)$ & $0.11(0.12)$ & $0.34 \mathrm{~ms}$ \\
\cline { 2 - 6 } & Amp. (SD) & $5.73(1.57)$ & $5.33(1.12)$ & $0.87(0.60)$ & $2.08 \mathrm{mV}$ \\
\hline
\end{tabular}

Table 2: Normal range of parameters in normal 20 subjects.

(PQ: Pronator Quadratus; CMAP: Compound Muscle Action Potential; FPL: Flexor Pollicis Longus; Amp.: Amplitude; SD: Standard Deviation)

PQ-CMAP) or supinated (recording FPL-CMAP). Palmar skin temperatures were not allowed to fall below 32. Observation was made using a Nicolet Viking electromyography system (Nicolet Instruments, Madison, WI, USA) and a 10-mm silver disc. CMAP from PQ and FPL were recorded with surface electrodes by supramaximal stimulation of the median nerve at the elbow. Stimulus duration was $0.1-0.2 \mathrm{~ms}$. In recording PQ-CMAP, the active electrode was placed over the dorsal side of the forearm at $5 \mathrm{~cm}$ proximal to the radial styloid, the reference electrode was over the radial styloid, and the ground electrode was over the forearm [20]. In recording FPL-CMAP, the active electrode was placed over the radial aspect on the volar one-third of the forearm, the reference electrode was over the volar side of the wrist and the ground electrode over the forearm [17] (Figure 1). PQ- and FPL-CMAP were recorded at the affected side and the normal side, and latency and the amplitude of the negative portion were analyzed. By the measurement of twenty healthy subjects, normal side-to-side differences in latency and amplitude were obtained as shown in Table 2. According to these criteria, we found delayed latency and low amplitude for PQ- and FPL-

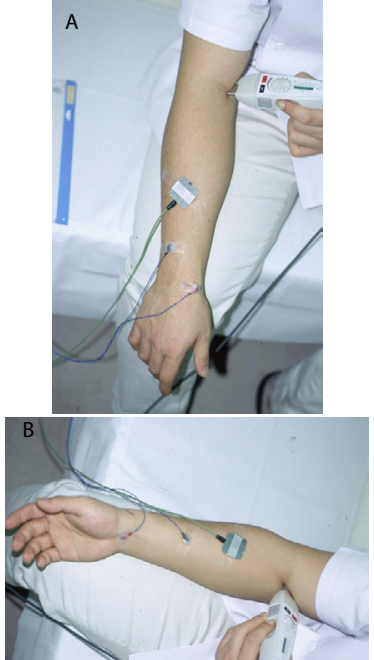

Figure 1: Methods of nerve conduction measurement for anterior interosseous nerve lesion.

A. PQ-CMAP was recorded using surface electrodes placed over the dorsal aspect of the distal forearm and median nerve stimulation was performed at the elbow. The active electrode was placed $5 \mathrm{~cm}$ proximal to the radial styloid, the reference electrode over the radial styloid, and the ground electrode proximal to the active electrode.

B. FPL-CMAP was recorded with the active electrode over the radial aspect on the volar one-third of the forearm, and the reference electrode over the wrist.

PQ: Pronator Quadratus, CMAP: Compound Muscle Action Potential, FPL: Flexor Pollicis Longus 
CMAP. The data were analyzed with the Student's t-test and the MannWhitney U test. A P-value less than 0.05 was considered statistically significant.

\section{Results}

In all 23 cases, both PQ- and FPL-CMAP were recorded initially, and all revealed abnormalities in PQ- and/or FPL-CMAP. Delayed latency and low amplitude were seen in 21 for PQ-CMAP and for FPL-CMAP in 17. Six cases showed only abnormal PQ-CMAP, two only abnormal FPL-CMAP and 15 indicated abnormality in both PQand FPL-CMAP. At the time of the final follow-up, an improvement in the strength of FPL, FDP1 and PQ was seen in 11 cases obtaining the British Medical Research Council (MRC) [25] grade 4 or better (complete recovery), whereas partial recovery in 11 and unchanged in one (Table 1). The onset of recovery of FPL, FDP1 or PQ from the onset of paralysis was two to 13 months (mean, 6 months) in 22 cases with complete or partial recovery. In case 7 , operative finding showed no adhesion or entrapment of AIN at the elbow and the forearm.

At the time of follow-up, 18 cases showed abnormal PQ- and/or FPL-CMAP, and five revealed normal parameters in both PQ- and FPLCMAP. The post-treatment PQ-CMAP latency shortened compared with the initial PQ latency in 16, unchanged in four and more delayed in three. The post-treatment PQ amplitude increased compared with the initial amplitude in 18, unchanged in three and decreased in two. The post-treatment FPL-CMAP latency shortened in 14, unchanged in three and more delayed in six. The post-treatment FPL amplitude increased in 14, unchanged in one, and decreased in eight.

The decrease in initial amplitude of PQ-CMAP showed a significant difference between the normal PQ strength group (-1.56) and the weak $\mathrm{PQ}$ strength group (-2.93), while the delayed latency of PQ-CMAP revealed no significance between the normal PQ and the weak PQ strength group (Table 3 ). There was a tendency that decreased amplitude of FPL-CMAP was larger in the weak FPL strength group $(-2.82)$ than that in the normal FPL strength group $(-0.2)$. There was no significant correlation between the initial PQ-CMAP or FPL-CMAP latency and final muscle strength, while there was a tendency that the sideto-side difference in amplitude of PQ- and FPL-CMAP in the partial recovery cases ( -2.85 and -3.27 , respectively) was larger than those in the complete recovery cases $(-1.76$ and -1.73 , respectively) (Table 4$)$.

\section{Illustrative Case}

A 69 year old female developed diffuse pain in the right elbow and the pain settled over four weeks. When she noticed weakness of pinch in the right hand, there were no sensory symptoms. Examination revealed weakness of the right FPL (MRC grade 4), FDP1 (2), and PQ (4), It also revealed temporal dispersion with $\mathrm{PQ}-\mathrm{CMAP}$ latency of $3.5 \mathrm{~ms}$ and

\begin{tabular}{|c|c|c|c|c|}
\hline & PQ: & $\begin{array}{c}\text { MRC: 2-4 } \\
\mathbf{( N = 1 2 )}\end{array}$ & $\begin{array}{c}\text { MRC: 5 } \\
\mathbf{( N = 1 1 )}\end{array}$ & p value \\
\hline \multirow{2}{*}{ PQ-CMAP } & Delayed latency (ms) (SD) & $0.85(0.95)$ & $0.33(0.35)$ & N.S. \\
\cline { 2 - 5 } & Decreased amp. (mV) (SD) & $-2.93(1.39)$ & $-1.56(1.26)$ & 0.05 \\
\hline \multirow{2}{*}{ FPL-CMAP } & FPL: & $\begin{array}{c}\text { MRC: 0-4 } \\
(\mathbf{N = 2 1 )}\end{array}$ & $\begin{array}{c}\text { MRC: 5 } \\
(\mathbf{N =} 2)\end{array}$ & \\
\cline { 2 - 5 } & Delayed latency (ms) (SD) & $0.45(0.99)$ & 0 & \\
\cline { 2 - 5 } & Decreased amp. (mV) (SD) & $-2.82(2.08)$ & -0.20 & \\
\hline
\end{tabular}

Table 3: The relationship between parameter (side-to-side difference) and MRC grade.

(PQ: Pronator Quadratus; MRC: Medical Research Council Grade; FPL: Flexor Pollicis Longus; CMAP: Compound Muscle Action Potential; amp.: amplitude; SD: Standard Deviation; N.S.: No Significance) amplitude of $0.8 \mathrm{mV}$, while the latency and amplitude on the normal side were $2.7 \mathrm{~ms}$ and $4.4 \mathrm{mV}$ (Figures $2 \mathrm{~A}$ and $2 \mathrm{~B}$ ). Recovery began after 13 months, and 19 months later, she indicated partial recovery of FPL (4), FDP1 (3), and PQ (5) of the right limb with PQ-CMAP latency and amplitude of $3.0 \mathrm{~ms}$ and $1.2 \mathrm{mV}$, respectively (Figure $2 \mathrm{C}$ ).

\begin{tabular}{|c|c|c|c|c|}
\hline & Recovery: & $\mathbf{P}, \mathbf{U}(\mathbf{N}=12)$ & $\mathbf{C ~ ( N = 1 1 )}$ & $\mathbf{p}$ value \\
\hline \multirow{2}{*}{ PQ-CMAP } & Delayed latency (ms) (SD) & $0.38(0.32)$ & $0.84(1.02)$ & N.S. \\
\cline { 2 - 5 } & Decreased amp. (mV) (SD) & $-2.85(1.15)$ & $-1.76(1.48)$ & N.S. \\
\hline \multirow{2}{*}{ FPL-CMAP } & Delayed latency (ms) (SD) & $0.20(0.28)$ & $0.68(1.32)$ & N.S. \\
\cline { 2 - 5 } & Decreased amp. (mV) (SD) & $-3.27(2.28)$ & $-1.73(1.70)$ & N.S. \\
\hline
\end{tabular}

Table 4: The relationship between parameter (side-to-side difference) and recovery (P: Partial Recovery; U: Unchanged; C: Complete Recovery (MRC grade 4 or better); PQ: Pronator Quadratus; CMAP: Compound Muscle Action Potential; FPL: Flexor Pollicis Longus; amp.: amplitude; SD: Standard Deviation; N.S.: No Significance)
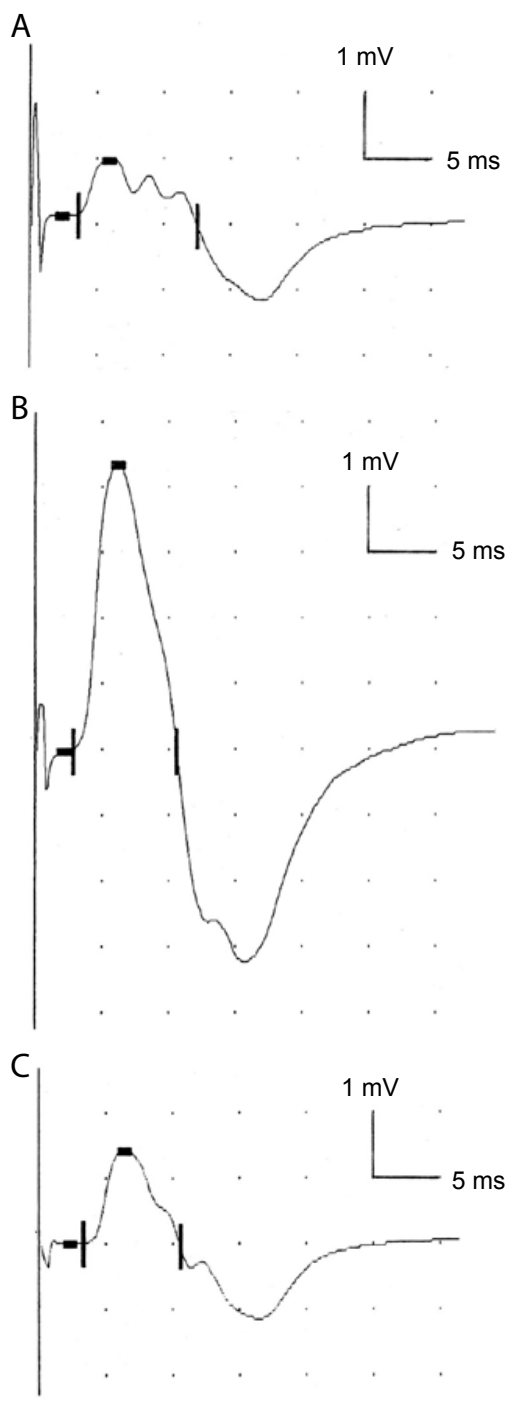

Figure 2: PQ-CMAP of a 69-year-old female with AIN lesion. A. Initially, latency was $3.5 \mathrm{~ms}$ and amplitude $0.8 \mathrm{mV}$, revealing temporal dispersion.

B. Latency and amplitude on the normal side were $2.7 \mathrm{~ms}$ and $4.4 \mathrm{mV}$. C. Nineteen months later, latency was $3.0 \mathrm{~ms}$ and amplitude $1.2 \mathrm{mV}$. 


\section{Discussion}

Anterior interosseous nerve (AIN) lesion with spontaneous onset remains a rare lesion and treatment should vary according to the etiology of the condition. Previous reports concerning the etiology of the AIN lesion are categorized into two, those describing the lesion as a compression, entrapment neuropathy and those describing it as a brachial plexus neuritis or neuralgic amyotrophy. Entrapment neuropathy is one of the causes of the AIN lesion. Structures that can compress the AIN are a tendinous origin of the deep head of the pronator teres or that of the flexor digitorum sublimis, enlarged bicipital bursae, thrombosed ulnar collateral vessels, the anomalous passage of the radial artery, and the supracondyloid process [4]. If no recovery occurs after conservative treatment of 6 to 12 weeks, surgical decompression should be performed, and the results of surgical treatment are generally good as reported in the literature [3-7,11,13,15]. However, MillerBreslow et al. [10] stated that surgical treatment did not affect recovery time. Meanwhile, in our study, one case (case 7) underwent surgical exploration with no demonstrable pathology detected, and gained partial recovery after 12 months from the onset of paralysis. On the other hand, Nagano [26] reported that a cause of the AIN lesion is hourglass-like fascicular constriction of the fasciculus of the median nerve destined to become the AIN distally, stating that interfascicular neurolysis leads to good results.

Another cause of the AIN lesion is a brachial plexus neuritis or neuralgic amyotrophy. Important points in the history to distinguish a brachial neuritis from local compression are the development of pain in the upper arm, elbow, or forearm that often precedes the motor paralysis, and an absence of trauma $[10,14]$. In our study, all cases showed nontraumatic onset of paralysis, with a high percentage of accompanying premonitory arm pain ( 20 of 23 patients: 87 percent), even without having shoulder girdle weakness. However, our findings suggest that these cases were suffering from brachial plexus neuritis [14]. Previous reports described conservative treatment with spontaneous recovery occurring up to 2 to 3 years after the onset of the palsy, and the results after conservative treatment are generally good $[2,8-10,12,14,16,18,21]$.

Electrodiagnosis is essential to the definite diagnosis of AIN lesions [21]. However a limited number of studies on nerve conduction have been reported [16-23]. Meya and Hacke [19] and Gardner-Thorpe [16] described PQ-CMAP latency with needle recording. Nakano et al. [18] and Seror [21] reported PQ-CMAP using needle recording to derive normative values. Nakano [18] reported the mean PQ-CMAP latency to be $5.1 \mathrm{~ms}$ ( 0.9 SD: standard deviation) whereas Seror [21] reported it to be $4.1(0.6) \mathrm{ms}$ with a side-to-side maximal latency difference of $0.8 \mathrm{~ms}$. Nakano [18] reported that five of seven patients showed abnormal latencies, and Seror [21] described that seven of 14 patients revealed abnormal latency and 12 of 14 patients showed low amplitude. However, recording electromyogram or PQ-CMAP using the needle electrode is painful for patients [18] and there is a risk of causing blood vessel injury. Mysiw and Colachis [20] reported PQCMAP using surface recording to develop normative values, stating that a mean latency was $3.5(0.4) \mathrm{ms}$ (left) and $3.6(0.4) \mathrm{ms}$ (right) with a side-to-side difference of $0.1(0.1) \mathrm{ms}$, and the amplitude of $3.1(0.8)$ $\mathrm{mV}$. Foley et al. [22] reported PQ-CMAP with surface recording from normal 207 subjects, stating that the latency was $4.2(0.5) \mathrm{ms}$, and the amplitude 4.3 (1.8) $\mathrm{mV}$. On the other hand, Craft et al. [17] reported FPL-CMAP using surface recording to derive normative values. Their optimal site for placing the active electrode was over the lateral distal one-third of the anterior surface of the forearm. They concluded that the latency of $4.0 \mathrm{~ms}$ and the amplitude of $2.5 \mathrm{mV}$ might serve as the limit of normal values. Vucic and Yiannikas [23] described FPL- CMAP using surface recording from 50 normal subjects, and their data were that the amplitude was $5.7(2.0) \mathrm{mV}$ (mean, 2SD) with side-to-side difference of $0.7(0.8) \mathrm{mV}$ and the latency was 3.9 (1.2) ms with side-toside difference 5.1 percent. They concluded that PQ- and FPL-CMAP using surface electrodes might aid diagnosis of AIN lesion.

Therefore, we recorded PQ-CMAP and FPL-CMAP using surface electrodes to study AIN lesions electrophysiologically according to Mysiw's method [20] and Craft's method [17]. The technique is simple and safe to perform and the information obtained is reproducible [20]. The criteria in our institute for the normal values of PQ-CMAP latency and amplitude (Table 2) were almost equal to those of Mysiw's and Foley's report, and the normal values of FPL-CMAP were also almost equal to those of Craft's and Vucic's report. There have been no reports about both PQ- and FPL-CMAP of AIN lesions. In our study, duration of paralysis was one to seven months, and seven months is a rather long for initial assessment and recovery might have begun during this time span, but four cases consulted our institute at the time of seven months after the onset of paralysis. All cases showed abnormality (delayed latency and/or low amplitude) in PQ- and/or FPL- CMAP. The posttreatment PQ latency shortened compared with the initial latency in 16 , the post-treatment PQ amplitude increased in 18, the post-treatment FPL latency shortened in 14 and the post-treatment FPL amplitude increased in 14 .

Concerning various patterns of neuropathic processes, segmental demyelination causes slowing of conduction across the affected segment, while axonal degeneration results in reduced amplitude of evoked potentials distally [27]. In our study, decreased amplitude in PQ-CMAP showed a significant difference between the normal PQ strength group and the weak PQ strength group, but delayed latency in PQ-CMAP revealed no significance between these two groups (Table 3). There was no significant correlation between the initial PQ- or FPLCMAP latency and the final muscle strength, while there was a tendency that the amplitude in side-to-side differences of PQ- and FPL-CMAP in partial recovery cases was larger than those in complete recovery cases (Table 4). These data of decreased amplitude indicates that an axonal degeneration in the motor fascicles to form the AIN is main pathology. The actual site of the neuritis has never been identified. Rennels and Ochoa [8] believe it to be proximal in the brachial plexus and England and Sumner [9] find the pattern more consistent with discrete lesions in multiple peripheral nerves. Rennels and Ochoa [8] described that the variability in the time for recovery could be explained on the basis of differing pathological processes in the nerve, stating that milder lesions with rapid recovery are probably seen with demyelination, while more severe lesions are a result of axonal degeneration that requires a longer period for axonal regrowth. In our series, the onset of recovery of FPL, FDP1, PQ from the onset of paralysis was an average of 6 months (range, two to 13 months) in 22 cases, which hints to the period of axonal regeneration from the proximal portion. Recently, Pham et al. [28] sought to determine the lesion site in 20 patients of spontaneous AIN lesions using high-resolution magnetic resonance neurography, and described that the AIN lesion in the majority of these cases was not a surgically treatable entrapment neuropathy but a multifocal mononeuropathy, which is selectively involved, within the main trunk of the median nerve, with the motor fascicles that continue distally to form the AIN. Our data suggest that an axonal degeneration in the proximal portion was main pathology and recovery began at the time of 6 months (average) from the onset of paralysis, therefore, we recommend non-surgical treatment for patients with spontaneous AIN lesions. 
Citation: Nobuta S, Sato K, Itoi E (2017) Retrospective Analysis of Anterior Interosseous Nerve Lesions and Motor Nerve Conduction Measurements. J Neurol Neurophysiol 8: 411. doi:10.4172/2155-9562.1000411

\section{Conclusion}

Recording and analysis of PQ- and FPL-CMAP were simple and safe, and were important in the definite electrodiagnosis of AIN lesions. The data suggest that an axonal degeneration in the proximal portion was main pathology of AIN lesions.

\section{Acknowledgement}

The authors wish to thank Ms. Yumi Watabe and Ms. Hiromi Takeda, Tohoku Rosai Hospital, for assisting in the manuscript preparation.

\section{References}

1. Parsonage MJ, Turner JW (1948) Neuralgic amyotrophy; the shoulder-girdle syndrome. Lancet 1: 973-978.

2. Kiloh LG, Nevin S (1952) Isolated neuritis of the anterior interosseous nerve. Br Med J 1: 850-851.

3. Fearn CB, Goodfellow JW (1965) Anterior interosseous nerve palsy. J Bone Joint Surg Br 47: 91-93.

4. Spinner M, Spencer PS (1974) Nerve compression lesions of the upper extremity. A clinical and experimental review. Clin Orthop Relat Res 104: 46-67.

5. Maeda K, Miura T, Komada T, Chiba A (1977) Anterior interosseous nerve paralysis. Report of 13 cases and review of Japanese literatures. The Hand 9: 165-171.

6. Rask M (1979) Anterior interosseous nerve entrapment: Kiloh-Nevin syndrome: Report of seven cases. Clin Orthop Relat Res 142: 176-81.

7. Hill NA, Howard FM, Huffer BR (1985) The incomplete anterior interosseous nerve syndrome. J Hand Surg Am 10: 4-16.

8. Rennels GD, Ochoa J (1980) Neuralgic amyotrophy manifesting as anterior interosseous nerve palsy. Muscle Nerve 3: 160-164.

9. England JD, Sumner AJ (1987) Neuralgic amyotrophy: An increasingly diverse entity. Muscle Nerve 10: 60-68.

10. Miller-Breslow A, Terrono A, Millender LH (1990) Non-operative treatment of anterior interosseous nerve paralysis. J Hand Surg Am 15: 493-496.

11. Schantz K, Riegels-Nielsen $P$ (1992) The anterior interosseous nerve syndrome. J Hand Surg Br 17: 510-512.

12. Goulding PJ, Schady W (1993) Favourable outcome in non-traumatic anterior interosseous nerve lesions. J Neurol 240: 83-86.
13. Sood MK, Burke FD (1997) Anterior interosseous nerve palsy. A review of 16 cases. J Hand Surg Br 22: 64-68.

14. Seki M, Nakamura $H$, Kono $H$ (2006) Neurolysis is not required for young patients with a spontaneous palsy of the anterior interosseous nerve. Retrospective analysis of cases managed non-operatively.J Bone Joint Surg $\mathrm{Br}$ 88: 1606-1609.

15. Ulrich D, Piatkowski A, Pallua N (2011) Anterior interosseous nerve syndrome: Retrospective analysis of 14 patients. Arch Orthop Trauma Surg 131: 1561-1565.

16. Gardner-Thorpe, C (1974) Anterior interosseous nerve palsy: Spontaneous recovery in two patients. J Neurol Neurosurg Psychiat 37: 1146-1150.

17. Craft S, Currier DP, Nelson RM (1977) Motor conduction of the anterior interosseous nerve. Phys Ther 57: 1143-1147.

18. Nakano KK, Lundergan C, Okihiro MM (1977) Anterior interosseous nerve syndrome: diagnostic methods and alternative treatments. Arch Neurol 34: 477-80.

19. Meya U, Hacke W (1983) Anterior interosseous nerve syndrome following supracondylar lesions of the median nerve: Clinical findings and electrophysiological investigations. J Neurol 229: 91-96.

20. Mysiw WJ, Colachis SC (1988) Electrophysiologic study of the anterior interosseous nerve. Am J Phys Med Rehabil 67: 50-54.

21. Seror P (1996) Anterior interosseous nerve lesions. Clinical and electrophysiological features. J Bone Joint Surg Br 78: 238-241.

22. Foley BS, Roslonski ET, Buschbacher RM (2006) Reference values for median nerve conduction to the pronator quadratus. Arch Phys Med Rehabil 87: 88-91.

23. Vucic S, Yiannikas C (2007) Anterior interosseous nerve conduction study: Normative data. Muscle Nerve 35: 119-121.

24. Nobuta $S$ (2008) Nerve conduction measurements for anterior interosseous nerve palsy. Clin Neurophysiol 36: 212-218 (in Japanese).

25. Mumenthaler M, Schliack H (1991) Peripheral nerve lesions: diagnosis and therapy. Thieme Medical Publishers, Inc., New York, p: 83.

26. Nagano A (2003) Spontaneous anterior interosseous nerve palsy. J Bone Joint Surg Br 85: 313-318.

27. Kimura J (1984) Principles and pitfalls of nerve conduction studies. Ann Neuro 16: 415-429.

28. Pham M, Baumer P, Meinck H, Schiefer J, Weiler M, et al. (2014) Anterior interosseous nerve syndrome. Fascicular motor lesions of median nerve trunk. Neurology 82: 598-606. 